\title{
Hydrophylicity Enhancement of Modified Cellulose Acetate Membrane to Improve the Membrane Performance in Produced Water Treatment
}

\author{
Tutuk Djoko Kusworo*, Danny Soetrisnanto, Cynthia Santoso, Tyas Dwi Payanti, and Dani Puji Utomo \\ Chemical Engineering Department, Faculty of Engineering, University of Diponegoro, Jl. Prof. Sudharto, Tembalang, Semarang, \\ 50239, Indonesia
}

\begin{abstract}
Produced water is a wastewater generated from petroleum industry with high concentration of pollutants such as Total Dissolved Solid, Organic content, and Oil and grease. Membrane technology has been currently applied for produced water treatment due to its efficiency, compact, mild and clean process. The main problem of produced water using membrane is fouling on the membrane surface which causes on low permeate productivity. This paper is majority focused on the improvement of anti-fouling performance through several modifications to increase CA membrane hydrophilicity. The membrane was prepared by formulating the dope solution consists of $18 \mathrm{wt} \%$ CA polymer, acetone, and PEG additive (3 wt$\%, 5 \mathrm{wt}-\%$, and $7 \mathrm{wt}-\%)$. The membranes are casted using NIPS method and being irradiated under UV light exposure. The SEM images show that parepared membrane has asymmetric structure consist of dense layer, intermediete layer, and finger-like support layer. The filtration test shows that PEG addition increase the membrane hydrophilicity and the permeate flux increases. UV light exposure on the membrane improves the membrane stability and hydrophilicity. The imrpovement of membrane anti-fouling performance is essential to achieve the higher productivity without lowering its pollutants rejection.
\end{abstract}

\section{Introduction}

Produced water is a by-product of the petroleum industry, where during production every barrel of obtained oil produces 3 barrels of produced water and will increase with the age of the oil well [1]. Produced water contains organic and inorganic materials such as Total Dissolved Solid (TDS) of 4380-49.987 mg/L, Magnesium 396.1-678 mg/L, Sulfate 6.2-621.3 mg/L, Calcium 987-2669 $\mathrm{mg} / \mathrm{L}$ [2]. In the condition of untreated produced water, it can affect the environment and human health [3].

Today, membrane technology is widely used for manufactured water treatment. Membrane technology is a beneficial technology because of its high separation efficiency and the addition of a small chemical [4]. In addition, the design of membrane equipment is also simple with low initial investment costs, low operational energy, and easy maintenance. The nanofiltration membrane is a type of membrane filtrations which effectively reduces the chemical content such as inorganic salts and small organic microsolutes present in produced water, and has a higher flux yield than the reverse osmosis membrane [5]. The nanofiltration membrane is a membrane having a pore size $<2 \mathrm{~nm}$.

Many studies have been performed to treat the produced water using membranes. Xi et al. [6] examined the surface-modified Poly (ether sulfone) membrane with electrophoresis - UV grafting, in which the amount of foulant can be reduced. Homayoonfal et al. [7] also modified the surface of the nanofiltration membrane by using UV-grafting Acylic acid on the surface of the Polysulfone membrane with the PEG additive where increasing surface irradiation time of the membrane will cause fouling decrease. However, those modification methods still produced fouled membranes. Fouling can cause a decrease in flux and shorten the life of the membrane used. Fouling on the membrane is the adsorption or accumulation of certain components in the produced water that occurs on the surface of the membrane (external fouling) or within the pores of the membrane (internal fouling).

According to Mondal and Wickramasinghe [8] and Alzahrani et al. [9], to overcome fouling, with increased hydrophilic properties in nanofiltration membranes can increase water permeability and increase rejection of both organic and inorganic components. In this study,

\footnotetext{
* Corresponding author: tdkusworo@che.undip.ac.id
} 
surface modification was done by mixing poly ethylene glycol additives (PEG). PEG plays a role in forming pores, increasing the number of pores on the nanofiltration membrane thus increasing membrane permeability, suppressing the amount of macrovoids and providing hydrophilic properties of the membrane. UV light exposure is also required to improve the performance and characteristic of cellulose acetate nonfouling nanofiltration membranes in treated water treatment.

\section{Materials and Methods}

\subsection{Materials}

The materials used in this research include: produced water from Pertamina Oil and Gas Company, Cirebon, cellulose acetate (CA) from MKR Chemicals, PEG 1000 and PEG 4000 from Sigma-Aldrich Chemie $\mathrm{GmbH}$ Steinheim Germany, 99.75\% Acetone from Mallinckrodt Chemicals, and distilled from UPT Diponegoro University Integrated Laboratory.

\subsection{Fabrication of Cellulose Acetate (CA) membranes}

Membrane preparation begins with the preparation of a polymer solution (casting) consisting of CA, PEG and Acetone polymers. The membrane was made with a fixed CA composition of $18 \mathrm{wt} \%$. The PEG types used were PEG 1000 and 4000, while the composition of variations of each type of PEG were $3 \mathrm{wt} \%, 5 \mathrm{wt} \%$, and $7 \mathrm{wt} \%$. The CA was inserted with Acetone into a closed erlenmeyer flask and stirred for 8 hours with a magnetic sirer until all the CA was dissolved, then PEG was added. Furthermore, the solution is allowed to stand for 1 day to remove air bubbles and ready to be printed by phase inversion technique.

The casting process was carried out by phase inversion technique, the dope solution was poured onto the glass plate that the edges are duct tape. The casting knife is then moved downward to form a thin layer on the glass plate, then irradiated with UV light with variations of time for 30,60 , and 90 seconds, then inserted into a coagulation bath containing aquades for 1 hour followed by immersion in another coagulation bath for 24 hours. Then the membrane is dried in a $40-50{ }^{\circ} \mathrm{C}$ oven for 24 hours.

\subsection{Membrane characterization}

\subsubsection{Flux and Rejection of fabricated membrane}

The flux value is determined a cross-flow filtration cell as shown in Figure 1. In the filtration cell is placed filter paper and membrane to be measured its permeability value. The distilled water at a volume of approximately $150 \mathrm{~mL}$ is fed into the filtration cell and tightly closed, then supplied with air pressure of 4 bar. The compacting process is carried out first in order to replace the polymer for 20-30 minutes. After the compaction process, the distilled water in the filtration cell is replaced by the water produced, the measurement of the produced water flux is carried out by measuring the volume of water produced which can be accommodated for a certain time interval at 15 minute intervals. The flux value is calculated by the ratio of permeate volume per unit time as in (Eq.1). The determination of the rejection coefficient is determined by determining the concentration before and after passing through the membrane as in (Eq.2).

$J=\frac{V}{P . A . t}$

$R=\left(1-\frac{C_{i}}{C_{0}}\right) \times 100 \%$

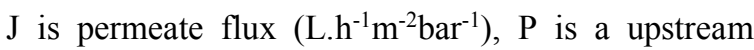
pressure (bar), $\mathrm{A}$ is an effective membrane area $\left(\mathrm{m}^{2}\right), \mathrm{t}$ is an operating time (second). $\mathrm{R}$ is a rejection efficiency, $\mathrm{C}_{\mathrm{i}}$ is the concentration of TDS in permeate, $\mathrm{C}_{0}$ is the concentration of TDS in feed.

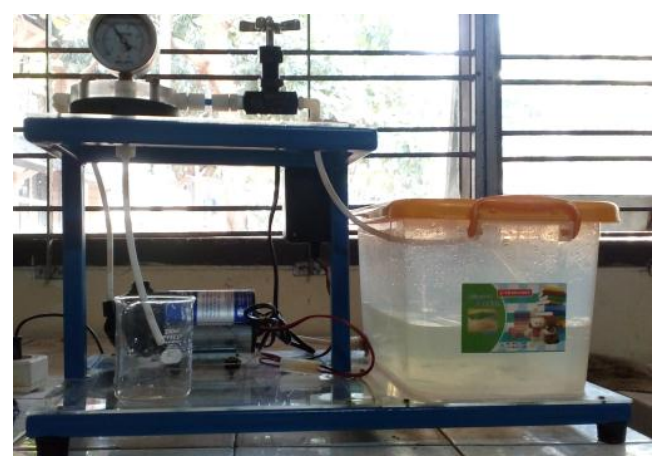

Figure 1. Membrane filtration cell

\subsubsection{Membrane structure morphology by Scanning Electron Microscopy}

The determination of membrane morphological structure is done by SEM (Scanning Electron Microscopy) with the following procedure. First the membrane is dried first, then the membrane is immersed in liquid nitrogen for a few seconds until it solidifies. Before the shooting, the membrane is lifted and broken both ends with tweezers. This piece of membrane is coated with pure gold (coating) that serves as a conductor. Furthermore, transverse sections and membrane surfaces are photographed with certain magnification.

\subsubsection{FTIR analysis (Fourier Transform Infra Red)}

Membrane characterization using FTIR was used to determine functional groups on the membrane. The test was performed to confirm the presence of cellulose acetate and PEG, as well as the influence of the type of PEG used and the irradiation of UV light on the membrane. 


\section{Results and Discussions}

\subsection{The effect of PEG Molecular Weight on membrane performance}

The performance of cellulose acetate membranes can be known from the membrane flux profile. Flux is the number of permeate volumes obtained at separation operations using membranes per unit area of membrane and time unit. Measurement of membrane flux is done by means of dead end filtration. Measurements and flux profiles of cellulose acetate membranes for treated water treatment with variations of the PEG additive type are shown in Figure 2.

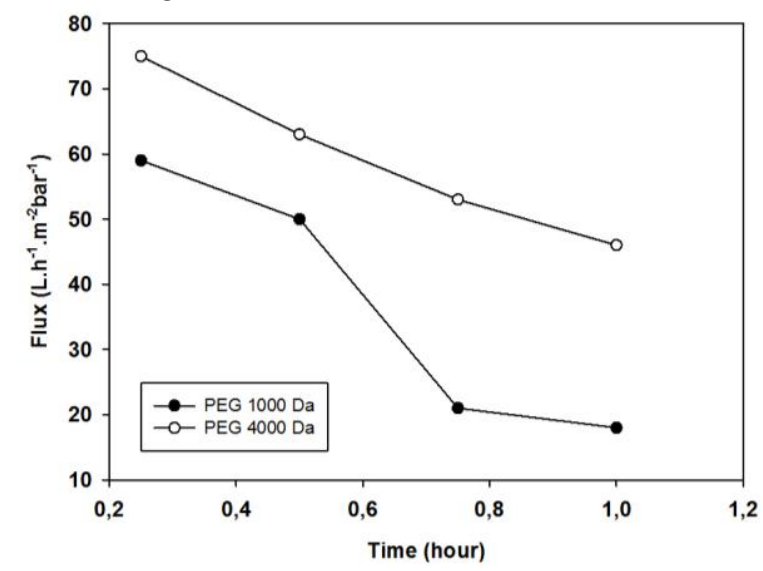

Figure 2. Fluxes profiles of fabricated membrane with different PEG molecular weights.

In the data presented in Figure 4.1. it can be seen that the flux will decrease over the time of filtration due to the fluctuation of the solute contained in the water produced on the surface of the membrane. The membrane with the PEG 4000 additive has a higher flux than the PEG 1000. This phenomenon corresponds to previous studies investigating the effects of 3 different PEG types (400 Da, $6000 \mathrm{Da}$ and 20,000 Da) on membrane transport and morphology [10]. PEG is an additive substance in the membrane that acts as a pore forming on the membrane and has hydrophilic properties. The molecular weight of PEG has an important role in the process of membrane formation rate. Increasing the molecular weight of PEG causes more PEG molecules trapped in the membrane due to reduced PEG mobility after immersion in the coagulation bath. At the same time, by increasing the molecular weight of PEG with the same concentration, the molecular quantity of PEG is decreased which will lead to the formation of larger pores, but fewer.

Membrane performance can also be determined by the value of membrane rejection. TDS rejection eficiency was determined by measurement of TDS concentration in both of upstream and downstream membrane filtration process. The effect of the PEG type on the TDS rejection efficiency is shown in Figure 3.

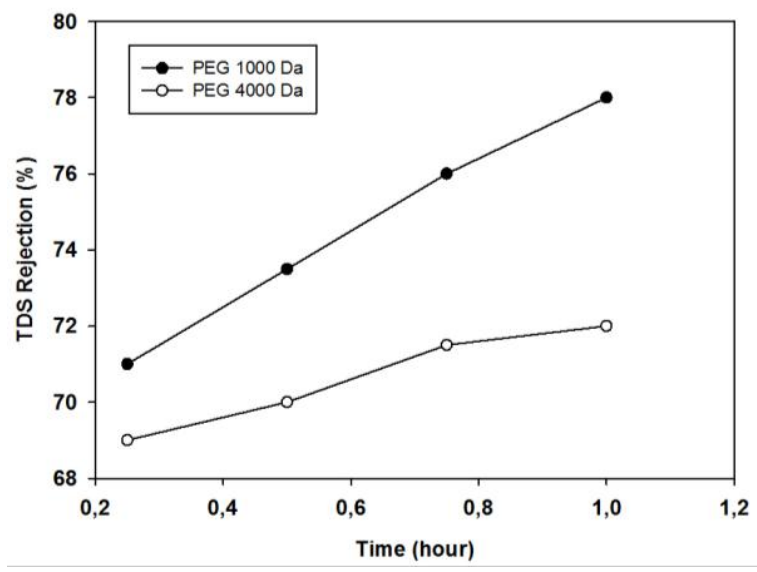

Figure 3. Rejection efficiencies of fabricated membranes with different PEG's MW

CA membrane with 1000 Da PEG doping has a higher TDS rejection efficiency than 4000 Da PEG doping. This could be due to the membrane with lower PEG additive has a lower membrane pore size. The PEG contributes as a porogent in membrane fabrication, the lower MW is well known gives smaller pore size.

\subsection{The effect of PEG concentration on CA membrane performance}

The flux profile of cellulose acetate membrane for treated water treatment with variation of PEG additive concentration is shown in Figure 4.

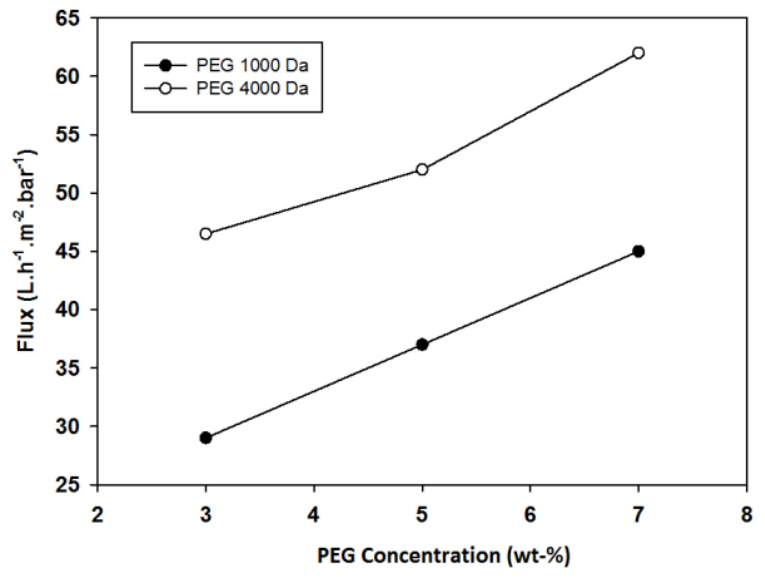

Figure 4. The average flux of membranes with various PEG concentration

The presence of PEG in the membrane can cause two effects: (1) The addition of PEG causes a decrease in solvent in the dope solution resulting in a comparison of polymer concentration with lower solvent concentration and an increase in the viscosity of the dope solution. Therefore, the dope solution becomes less stable, resulting in instantaneous demixing when the dope solution mold is immersed in the coagulation bath. Instantaneous demixing means that the membrane will soon form when the dope solution mold is inserted into a non-solvent coagulation bath. (2) PEG has a natural hydrophilic property. This hydrophilic property helps the exchange of water as non-solvent with acetone in the 
membrane during the phase inversion process and affects the speed of membrane formation and membrane morphology [11].

PEG acts as a pore-forming that is completely mixed in dope solution and will dissolve with water during the coagulation process. When PEG dissolves in water, the pores begin to form into finger-shaped structures or macrovoid holes. Increased PEG concentrations will lead to an increase in the number of pores on the membrane, so the resulting flux will increase as well.

The values of flux and rejection are directly related to the amount of pore and pore mass of the membrane. From the data presented in Figure 4 shows that the greater the concentration of PEG added to the dope solution the greater the membrane flux obtained. One of the most important things in membrane applications is the ability of membranes to reject certain solutes. In this study, rejection test was conducted on TDS concentration. The data of membrane test results in rejecting the solute are shown in Figure 5.

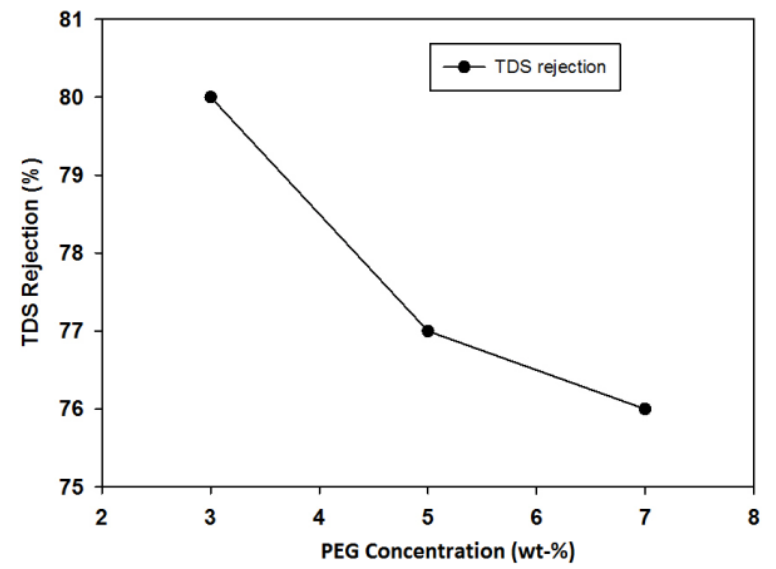

Figure 5. TDS rejection profile of CA membranes with various PEG concentration

In Figure 5, it is seen that the more PEG concentrations are added the rejection will decrease. This is because the more PEG concentrations added to the dope solution will increase the amount of pore [12]. PEG is an additive substance that is hydrophilic and easily soluble with water. The PEG mixed in the dope solution will dissolve in water at the membrane coagulation stage so that it will cause macrovoid in the membrane structure. The greater the percentage of PEG in dope solution, will lead to more pores. If the pores on the membrane are more numerous, the solute present in the produced water will have a chance of escaping during filtration causing a decrease in the membrane rejection value.

\subsection{The effects of UV irradiation on CA membrane performance}

UV irradiation on the membrane is a pre-treatment prior to the coagulation process. The effect of UV treatment on the membrane can be determined by comparing the membrane performance with UV treatment and without
UV treatment on the same type and PEG concentration as shown in Figure 6

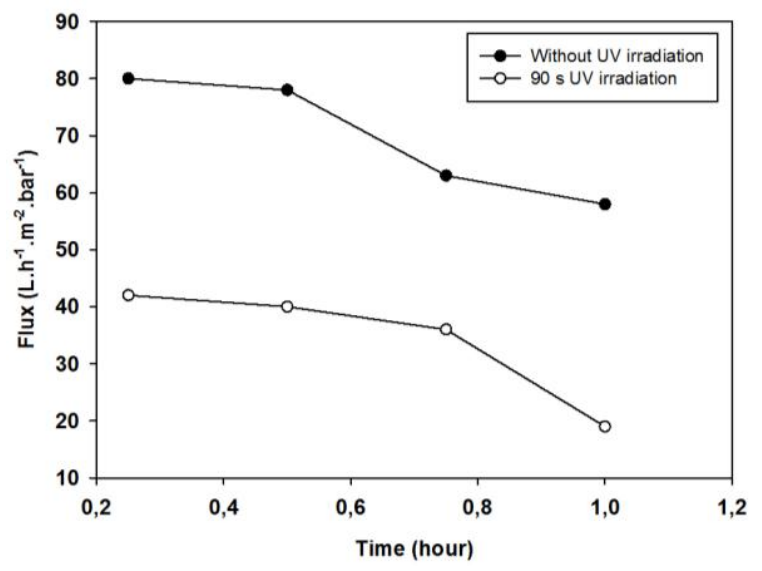

Figure 6. Flux profiles of UV irradiated membranes

Figure 6 shows the performance of cellulose acetate membrane flux with PEG 1000 of $7 \%$ without UV irradiation and with UV irradiation for 90 seconds. Membrane with UV irradiation treatment has a lower flux result. The resulting lower flux results from changes in the membrane structure caused by UV light exposed on the membrane surface.

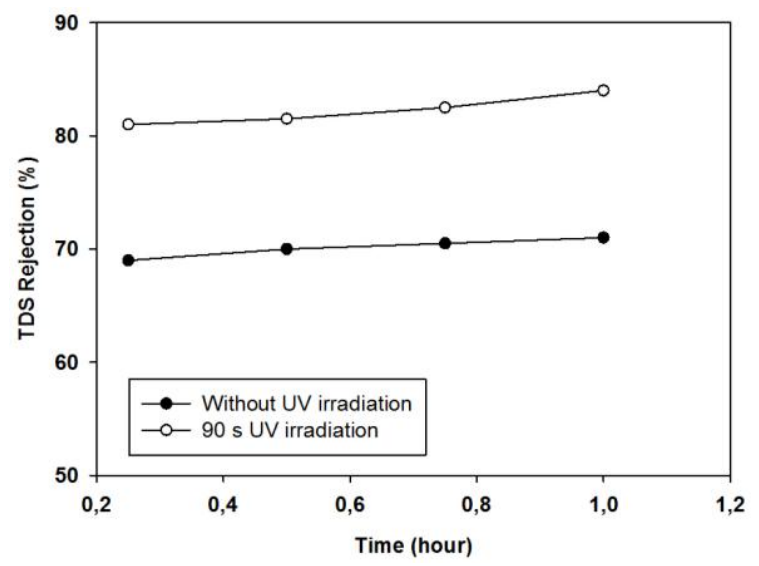

Figure 7. TDS rejection efficiency of UV irradiated CA membrane

Membrane without UV irradiation has a larger flux, but when viewed with regard to the rejection value of the membrane, the UV membrane has a better rejection value. Rejection profiles of Total dissolved solid (TDS) is shown in Figure 7. UV irradiation on the membrane serves for chain scission and crosslinking [13]. Furthermore, UV rays will cause polymerization of the membrane. Polymerization occurs due to the formation of free radicals in the polymer due to UV irradiation, which in turn decreases pore size and increases the hydrophilic properties of the membranes.

\subsection{Membrane Characteristics}

\subsubsection{Membrane structure morphology}

The membrane struture prophology was observed using Scanning electron microscopy (SEM). Figure 8 shows the images of surface and cross-sectional membrane structures. 


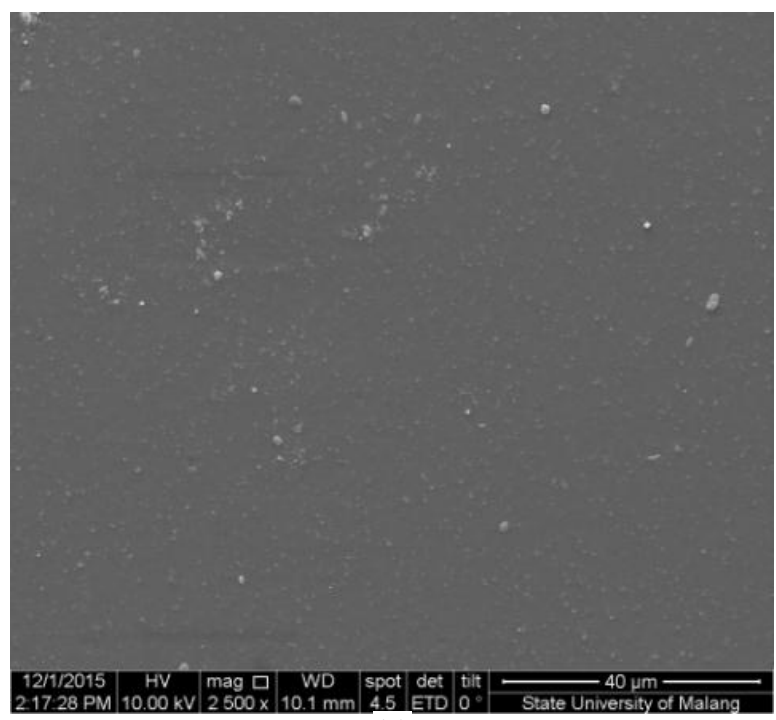

(a)

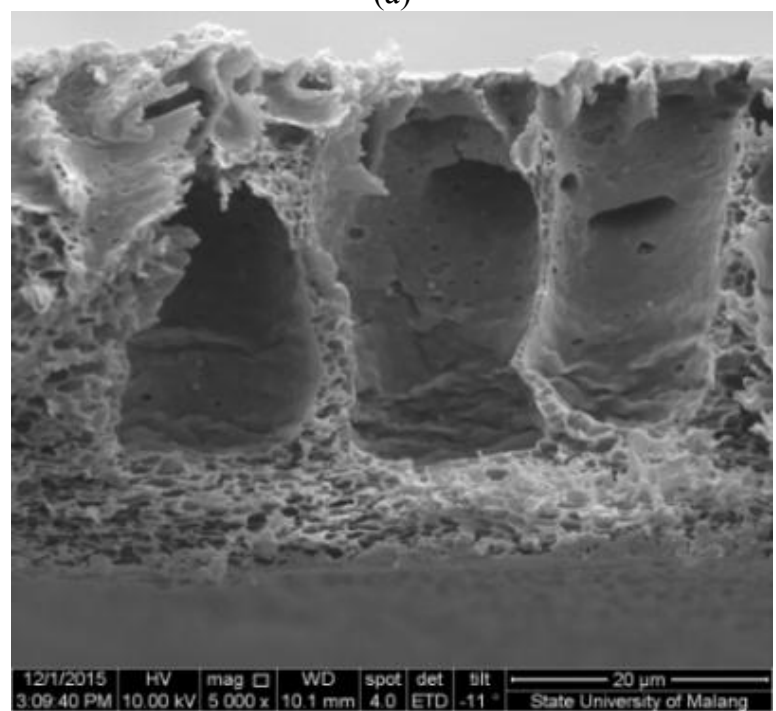

(b)

Figure 8. SEM images of fabricated membrane (a) surface section, (b) cross-section.

Figure 8.a shows a flat smooth membrane surface of CA membrane. At this magnification $(5.000 \mathrm{x})$, no voids were observed. The white spot in the membrane surface could be due to the agglomeration of CA polymer. This phenomenon occured because of un-spontaneous demixing in whole flat sheet membrane during coagulation process in non-solvent soaking. The Figure 8.b shows a finger-like porous structure of crosssectional membrane. This structure contributes as a support and maintain the permeability of membrane. The membrane consists of three layer: top layer is a dense layer which plays an important role in selectivity performance, the intermediate layer is a porous layer which acts as support and maintaining the membrane flux.

\subsubsection{FTIR analysis}

Characterization of cellulose acetate membranes using FTIR analysis was performed to determine the functional groups present in the membrane. Figure 8 shows the FTIR characterization of a 7\% PEG 1000\% cellulose acetate membrane with variation without UV irradiation and with UV irradiation for 90 seconds.

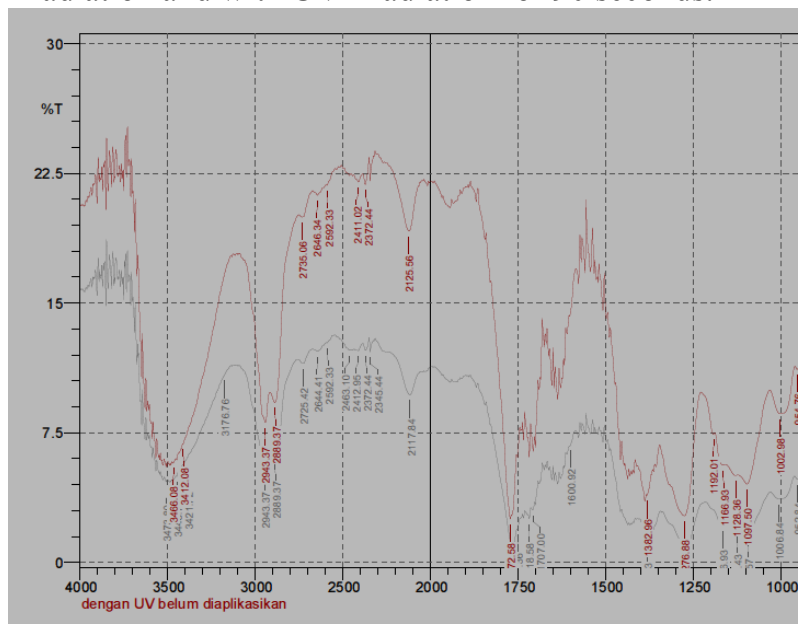

Figure 9. FTIR spectra of Unirradiated and UV irradiated CA membranes.

FTIR spectra showed that cellulose acetate membrane has $\mathrm{C}-\mathrm{O}-\mathrm{C}-\mathrm{CH}_{3}-\mathrm{COOH}$ and $\mathrm{C}=\mathrm{O}$ groups. Cellular cellulose acetate membranes with UV irradiation have higher transmittance values in all clusters compared with UV-free membranes. The C-O-C group on the UV less membrane lies at a wavelength of $1095.57 \mathrm{~cm}^{-1}$ and on the UV irradiated membrane lies at a wavelength of $1097.50 \mathrm{~cm}^{-1}$. While the $\mathrm{C}=\mathrm{O}$ group on the membrane without UV lies at wavelength 1751.36 and on the membrane with UV irradiation located at wavelength $1772.58 \mathrm{~cm}^{-1}$. The occurrence of these wavelength shifts indicates that UV irradiation treatment effect on membrane morphology structure.

In membrane treatment with UV irradiation, there are chain scission, crosslinking and then polymerization process on the membrane. This is illustrated in Figure 9, which is the FTIR result of two membranes without and with UV treatment for 90 seconds. Appears peak at the wave number $1050-1300 \mathrm{~cm}^{-1}$ which may indicate the presence of a C-O group of Alcohol / ether / carboxylic acid / ester usually present in the wave number. Appears on a wave number $2500-2700 \mathrm{~cm}^{-1}$ which may indicate the presence of an $\mathrm{O}-\mathrm{H}$ group of carboxylic acids with the hydrogen bonds normally present in the wave number. Appears on top of the wave number 3200-3600 $\mathrm{cm}^{-1}$ which may indicate the presence of an $\mathrm{O}-\mathrm{H}$ group of hydrogen / phenol bonding alcohols which usually appear on the wave number.

There is a wide difference of peaks on both membranes where FTIR results from cellulose acetate membranes treated with UV light irradiation have larger area area than non-UV irradiated membrane. This wide area difference is due to the denser membrane structure. The denser structures are formed because the interactions between molecules of the membrane become larger due to UV irradiation resulting in the absorbance of the membrane against Infrared light from FTIR equipment that increases significantly. 


\section{Conclusion}

Cellulose-based nanofiltration membranes have been successfully manufactured. The cellulose acetate nanofiltration membrane is an asymmetric membrane consisting of three layers of dense layer, intermediate layer and support layer. The filtration test shows that PEG addition increase the membrane hydrophilicity and the permeate flux increases. UV light exposure on the membrane improves the membrane stability and hydrophilicity. The imrpovement of membrane antifouling performance is essential to achieve the higher productivity without lowering its pollutants rejection.

The authors would thank to Waste treatment laboratory of Chemical Engineering Department UNDIP for supporting facilities.

\section{References}

1. F.R. Ahmadun, A. Pendashteh, L.C. Abdullah, D.R.A. Biak, S.S. Madaeni, Z.Z. Abidin, J. Hazard. Mater. 170, 530-551 (2009).

2. S. Alzahrani, A.W. Mohammad, N. Hilal, P. Abdullah, O. dan Jaafar, Sep. Purif. Technol. 118 324-341 (2013).

3. H.I. Safitri, F. Ryanitha, and N. Aryanti, J. Teknol. Kimia Industri, 2 (4), 205-211 (2013).

4. M.J. Han, and S.T. Nam, J. Membr. Sci. 202, 55-61 (2002)

5. A.W. Mohammad, Y.H. Teow, W.L. Ang, Y.T. Chung, D.L.O. Radcliffe, N. Hilal, Desalination. 356, 226-254 (2014).

6. W. Xi, W., Rong, L. Zhangsheng, A.G. Fane, J. Membr. Sci. 273, 47-57 (2006).

7. M. Homayoonfal, A. Akbari, and M.R. Mehrnia, Desalination, 263, 217-255 (2010).

8. S. Mondal, and S.R. Wickramasinghe, J. Membr. Sci. 322, 162-170 (2008).

9. S. Alzahrani, A.W. Mohammad, N. Hilal, P. Abdullah, and O. Jaafar, J. Environ. Chem. Eng., 1, 1341-1349 (2013).

10. B. Chakrabarty, A.K.A Ghoshall, and M.K. Purkait., J. Membr. Sci. 309, 209-221 (2008).

11. Y. Ma, F. Shi, J. Ma, M. Wu, J. Zhang, and C. Gao, Desalination. 272, 51-58 (2011).
12. T.D. Kusworo, Budiyono, J. Supriyadi and D.C. Hakika. Inter. J. Waste Resource, 4(1), 1-9 (2014).

13. N. Addina, K.S. Dini, and S. Heru, J. Teknol. Kimia Industsri, 2(4), 189-197 (2013). 